\title{
Rate-and-State Theory of Plastic Deformation Near a Circular Hole
}

\author{
J. S. Langer \\ Department of Physics, University of California, Santa Barbara, CA 93106 \\ Alexander E. Lobkovsky \\ Institute for Theoretical Physics, University of California, Santa Barbara, CA 93106
}

(May 6, 2019)

\begin{abstract}
We show that a simple rate-and-state theory accounts for most features of both time-independent and time-dependent plasticity in a spatially inhomogeneous situation, specifically, a circular hole in a large stressed plate. Those features include linear viscoelastic flow at small applied stresses, strain hardening at larger stresses, and a dynamic transition to viscoplasticity at a yield stress. In the static limit, this theory predicts the existence of a plastic zone near the hole for some but not all ranges of parameters. The rate-and-state theory also predicts dynamic failure modes that we believe may be relevant to fracture mechanics.
\end{abstract}

\section{INTRODUCTION}

Since the work of Hart [1] in the 1960's, scientists have understood that a satisfactory theory of plastic deformation in solids must include dynamic variables that describe the internal states of materials. The deformation field itself cannot be sufficient. It cannot, in any natural way, describe the irreversible changes that lead to hysteretic stressstrain curves, or to the transition from nonlinear viscoelastic to viscoplastic behavior with increasing applied stress. Conventional theories of plasticity cope with these limitations by specifying phenomenological rules to suit various situations and histories of deformation. For example, strain hardening curves, viscoplastic laws, or the distinctions between loading and unloading behaviors are determined from experiment and used as needed in computations. In most treatments there is also a sharp distinction between time-independent and time-dependent behaviors, with little or no indication of how these properties may be related to one another. We believe that a deeper, more nearly fundamental level of phenomenology is required for modern applications, for example, for computing deformations near moving crack tips. Plasticity is an intrinsically time dependent phenomenon; time-independent descriptions should emerge as static limits of fully dynamic theories.

In a recent paper [2], M. Falk and one of the present authors (JSL) proposed a theory of plastic deformation in amorphous solids in which they introduced an internal state variable to describe the orientations of what they called "shear-transformation zones." (We refer to this as the "STZ" theory.) The resulting "rate-and-state" equations (a concept that is widely used in the seismological literature [3, 1 ] and in recent theories of friction [5]) successfully describe the full range of viscoelastic and viscoplastic phenomena, including hysteretic effects. The basic structure of the STZ theory appears to be broadly applicable. The new internal state variable might equally well describe, for example, anisotropy in the way dislocations pile up near defects in crystalline materials.

Reference [2] discusses plasticity only in spatially uniform situations. Our purpose here is to apply a simple version of the STZ theory to a spatially inhomogeneous situation and to make contact with conventional plasticity theory. We especially want to learn whether the conventional picture of a time-independent plastic zone appears in the static limit. Looking ahead to fully dynamic situations such as fracture, we also want to understand the dynamics of plastic flow in regions of concentrated stress. We shall show that the conventional time-independent concepts - the "yieldsurface" hypotheses - do emerge from dynamic theories in an approximate way in many normal situations. As we shall argue, however, the rate-and-state theory is simpler, richer and more general than the conventional approaches.

The problem of describing spatially inhomogeneous plastic deformation is best approached by looking at a simple example where questions of time dependence and compatibility are not obscured by mathematical details. A growing circular hole in a very large stressed plate satisfies the criterion of simplicity. It shares important features with the case of plastic deformation near a crack tip; the tractions are applied at a distance and the stresses are concentrated near the hole. Several researchers have addressed the problem of dynamic hole growth in the context of ductile fracture [6] and spallation [7]. Carroll and Holt and later Johnson included inertial effects but neglected rate dependent plasticity [8.6]. Bodner and Partom used a version of a conventional rate dependent plasticity theory but did not study a stress controlled situation [9].

The scheme of this paper is as follows. In Section II, we derive equations of motion for the radius of the circular hole and the surrounding stress field, assuming a general form for the constitutive relation that governs the rate of plastic deformation. Section III contains a brief summary of several results of conventional plasticity theory which will serve as points of comparison for the rate-and-state analysis. A simplified version of the STZ model is introduced in Section 
IVA where, for completeness, we outline some important properties of this model that were reported previously in [2] and [10]. We describe the dynamic behavior of the STZ model for the hole problem in Section IVB. The paper concludes with remarks about the implications of these results in Section $\mathrm{V}$.

\section{DYNAMIC PLASTICITY IN A CIRCULAR GEOMETRY}

Throughout this analysis, we consider only a two-dimensional solid in a state of plane strain, and assume that inertial effects are negligible. Suppose that a circular hole in this system has radius $R(t)$ at time $t$. Outward tractions at the distant edges of the plate cause the pressure $p$ far from the hole to be $p \rightarrow-\sigma_{\infty}$. We introduce polar coordinates $r$ and $\theta$ that define an Eulerian reference frame, so that $u(r, t)$ is the radial displacement, measured from some initial reference state, of the material currently at position $r$. The function $u(r, t)$ is the only degree of freedom in the problem.

The total rate-of-deformation tensor (including both elastic and plastic parts) is diagonal with components:

$$
\mathcal{D}_{r r}^{t o t}=\frac{\partial v}{\partial r} ; \quad \mathcal{D}_{\theta \theta}^{t o t}=\frac{v}{r}
$$

where $v$ is the material velocity:

$$
v=\frac{\partial u / \partial t}{1-\partial u / \partial r}
$$

For small strains, the tensor $\mathcal{D}^{\text {tot }}$ is approximately equal to the total strain-rate tensor $\dot{\varepsilon}^{\text {tot }}$. Eq.(2.1) implies that the components of $\mathcal{D}^{t o t}$ satisfy the compatibility condition:

$$
\frac{\partial}{\partial r}\left(r \mathcal{D}_{\theta \theta}^{t o t}\right)=\mathcal{D}_{r r}^{t o t}
$$

The stress tensor has the form:

$$
\sigma_{r r}=-p-s, \quad \sigma_{\theta \theta}=-p+s .
$$

Here, $s=\left(\sigma_{\theta \theta}-\sigma_{r r}\right) / 2$ is the deviatoric stress. The elastic stress-strain relations are:

$$
2 \mu \varepsilon_{r r}^{e l}=-(1-2 \nu) p-s, \quad 2 \mu \varepsilon_{\theta \theta}^{e l}=-(1-2 \nu) p+s,
$$

where $\mu$ is the shear modulus and $\nu$ is Poisson's ratio. In the absence of inertial effects, balance of forces implies

$$
\frac{\partial p}{\partial r}=-\frac{1}{r^{2}} \frac{\partial}{\partial r}\left(r^{2} s\right) .
$$

The boundary condition at $r=R$ is

$$
-\sigma_{r r}(R, t)=p(R, t)+s(R, t)=0 .
$$

For simplicity, we neglect surface tension. Also at $r=R$, we have

$$
\frac{\dot{R}}{R}=\mathcal{D}_{\theta \theta}^{t o t}[s(R), \ldots] .
$$

Here and elsewhere, dots above symbols denote derivatives with respect to time $t$.

For incompressible plasticity, the constitutive relation has the form:

$$
\mathcal{D}_{\theta \theta}^{p l}=-\mathcal{D}_{r r}^{p l} \equiv \mathcal{D}(s, \ldots),
$$

where $\mathcal{D}$ is some function of $s$ and possibly other variables as indicated by the ellipsis. Combining (2.9) with the compatibility condition (2.3), the elasticity equations (2.5), and force balance (2.6), we find

$$
\mathcal{D}(s, \ldots)+\frac{(1-\nu)}{\mu} \dot{s}=\frac{C(t)}{r^{2}},
$$


where $C(t)$ is an $r$-independent constant of integration. Further analysis using the boundary conditions (2.7) and (2.8) at $r=R$ yields

$$
C(t)=\left[1+\left(\frac{1-2 \nu}{\mu}\right) s(R, t)\right] R \dot{R}
$$

and

$$
\left[1-\frac{1}{\mu} s(R, t)\right] \frac{\dot{R}}{R}=2 \int_{R}^{\infty} \frac{d r}{r} \mathcal{D}[s(r, t), \ldots] .
$$

Eqs. (2.10) through (2.12), supplemented by equations of motion for other arguments of $\mathcal{D}(s, \ldots)$, constitute a coupled set of first-order differential equations suitable for computing the time evolution of $s(r, t)$ and $R(t)$.

If $R(t)$ is the only length scale in the problem, we can look for self-similar solutions in which $\dot{R} / R=\omega=$ constant so that the hole radius is growing exponentially. All functions depend only on $\xi=r / R(t)$. Combining the expression for $C(t)$ in (2.11) with (2.10), and transforming to the scaling variable $\xi$, we find

$$
\mathcal{D}(\tilde{s}, \ldots)-\frac{(1-\nu)}{\mu} \omega \xi \frac{d \tilde{s}}{d \xi}=\frac{\omega}{\xi^{2}}\left[1+\left(\frac{1-2 \nu}{\mu}\right) \tilde{s}(1)\right] .
$$

Force balance (2.6) plus the boundary condition (2.7) imply

$$
\sigma_{\infty}=2 \int_{1}^{\infty} \frac{d \xi}{\xi} \tilde{s}(\xi)
$$

If these self-similar solutions exist for $\omega>0$, they describe unbounded plastic failure of the material.

\section{CONVENTIONAL THEORIES}

In a typical time-independent approach to this problem [11], one assumes that there exists a maximum value of $s$, say $s_{y}$, and that, in any sufficiently slow deformation, the material adjusts its state so that $s \leq s_{y}$ everywhere. Technically, the condition $s=s_{y}$ is a special case of a Tresca yield surface in the space of stress components. For present purposes, we take this assumption to mean that the hole is surrounded by a plastic zone, $R<r<R_{1}$, within which the force-balance equation (2.6) remains valid but the condition $s=s_{y}$ replaces Hookean elasticity (2.5). Outside this zone, $r>R_{1}, p=-\sigma_{\infty}$ and $s=s_{y} R_{1}^{2} / r^{2}$. Continuity of stress at $R_{1}$ means that, within the zone, $p=-\sigma_{\infty}-2 s_{y} \ln \left(r / R_{1}\right)$. Then the boundary condition (2.7) at $r=R$ implies that

$$
\ln \frac{R_{1}}{R}=\frac{1}{2}\left(\frac{\sigma_{\infty}}{s_{y}}-1\right)
$$

Thus, these assumptions predict that a stationary state with a non-vanishing plastic zone exists for $\sigma_{\infty}>s_{y}$. A calculation of the displacements similar to that described by Hill [12] shows that the hole radius $R$ diverges as $\sigma_{\infty}$ approaches an upper threshold stress $\sigma_{\infty}^{t h}$ which (for the case $s_{y} / \mu \ll 1$ ) is given by:

$$
\frac{\sigma_{\infty}^{t h}}{s_{y}}=1+\ln \left(\frac{\mu}{2 s_{y}(1-\nu)}\right) \text {. }
$$

To see what happens above this stress, we must consider a time-dependent theory.

The simplest conventional time-dependent hypothesis is the Bingham law which, for $s \geq 0$, we can write in the form:

$$
\mathcal{D}(s)= \begin{cases}\alpha\left(s-s_{y}\right) & \text { for } s \geq s_{y} \\ 0 & \text { otherwise }\end{cases}
$$

where $\alpha$ is a response coefficient. Essentially by definition, the combination of (3.3) with (2.10)-(2.12) describes an elastic perfectly-plastic material whose stationary states, for $s_{y}<\sigma_{\infty}<\sigma_{\infty}^{t h}$, are the same as those described in the preceding paragraph. We have checked that these states are stable attractors by using (3.3) to integrate (2.10)-(2.12), using the initial condition $s(r, 0)=\sigma_{\infty} R^{2}(0) / r^{2}$ (for $r>R(0)$ ). We found no surprises; a plastic zone consistent with (3.1) forms around the growing hole. 
We also can compute the self-similar solutions of 2.13 ) for the Bingham model. For ease of analysis, we write these for the case of incompressible elasticity, $\nu=1 / 2$. In the outer, elastic region, $\xi>\xi_{1}=\left(\mu / s_{y}\right)^{1 / 2}$ :

$$
\tilde{s}(\xi)=s_{y}\left(\frac{\xi_{1}}{\xi}\right)^{2}
$$

and, in the plastic region, $s \geq s_{y}, 1<\xi<\xi_{1}$ :

$$
\tilde{s}(\xi)=s_{y}+\left(\frac{\mu \omega}{\mu \alpha+\omega}\right) \frac{1}{\xi^{2}}\left[1-\left(\frac{\xi}{\xi_{1}}\right)^{\beta+2}\right],
$$

where $\beta(\omega)=2 \mu \alpha / \omega$. Note that the exponent $\beta$ becomes indefinitely large in the limit of small $\omega$; thus the second term in the square brackets in (3.5) produces a function $\tilde{s}(\xi)$ that is sharply bent but continuous at $\xi=\xi_{1}$. To complete the calculation, we use (3.5) to evaluate the right-hand side of (2.14). After rearranging and taking the limit of small $\omega$, we find:

$$
\omega \approx \frac{\alpha \mu}{s_{y}}\left(\sigma_{\infty}-\sigma_{\infty}^{t h}\right)
$$

where $\sigma_{\infty}^{t h}$ is the upper threshold defined in (3.2). Thus, as expected, the dynamic failure modes start where the time-independent theory breaks down.

\section{THE STZ MODEL}

\section{A. Basic properties}

For present purposes, it will be sufficient to use the simplified version of the STZ model that we introduced in an earlier one-dimensional analysis [10]. As in the Bingham case, the model is specified by the function $\mathcal{D}(s, \ldots)$ defined in (2.9). For two-dimensions, with circular symmetry, we write:

$$
\mathcal{D}(s, \Delta)=\frac{1}{\tau}(\lambda s-\Delta)
$$

and supplement this by an equation of motion for the state variable $\Delta$ :

$$
\dot{\Delta}=\mathcal{D}(s, \Delta)(1-\gamma s \Delta) \text {. }
$$

$\Delta(r, t)$ is the single independent element of a diagonal, traceless tensor which describes the local anisotropy of the shear-transformation zones. We are omitting the other state variable in [2] that describes the density of STZ's on the assumption that this quantity quickly reaches its equilibrium value. More importantly, this simplified version of the STZ model omits the strongly $s$-dependent rate factor that governs memory effects. This version is qualitatively sensible if we load the system only once in one direction, but it does not behave properly if the loading is cycled in any way.

The inverse stress $\gamma$ can be eliminated in favor of a group of parameters that plays the role of a dynamic yield stress, specifically, $s_{y}=1 / \sqrt{\lambda \gamma}$. Note what is happening here for spatially uniform situations. For $s<s_{y}, \Delta(t)$ has its stable fixed points on the line $\Delta=\lambda s$, where $\mathcal{D} \cong \dot{\varepsilon}_{\theta \theta}^{p l}=0$. In this region, the material is nonlinearly viscoelastic. For $s \ll s_{y}$ or, equivalently, $\gamma \rightarrow 0$, it obeys a conventional creep-compliance law [10]:

$$
\varepsilon^{t o t}(t)=(1+\lambda) s(t)-\lambda \int_{-\infty}^{t} d t^{\prime} \exp \left[-\frac{1}{\tau}\left(t-t^{\prime}\right)\right] \dot{s}\left(t^{\prime}\right) .
$$

We obtain a particularly important result by supposing that the system is initially in a state with $\varepsilon_{\theta \theta}^{p l}=\Delta=0$ and that a stress $s<s_{y}$ is applied suddenly at time $t=0$. A simple calculation then yields:

$$
\varepsilon_{\text {final }}^{p l} \equiv \varepsilon_{\theta \theta}^{p l}(t \rightarrow \infty)=-\frac{\lambda s_{y}^{2}}{s} \ln \left(1-\frac{s^{2}}{s_{y}^{2}}\right)
$$

$\varepsilon^{p l}(t)$ approaches $\varepsilon_{\text {final }}^{p l}$ exponentially in time with a relaxation time $\tau_{\text {relax }}$ that diverges as $s \rightarrow s_{y}$ : 


$$
\tau_{\text {relax }}=\frac{\tau}{1-\left(s / s_{y}\right)^{2}}
$$

Eq. (4.4) is a strain-hardening curve; that is, $\varepsilon_{\text {final }}^{p l}$ is the non-recoverable plastic strain produced after an infinitely long time by the deviatoric stress $s$. In the limit $\lambda \rightarrow 0$, with $s_{y}$ held constant, $\varepsilon_{\text {final }}^{p l}$ vanishes for $s<s_{y}$ but can have any positive value for $s \cong s_{y}$. Thus the parameter $\lambda$ is a measure of the deviation from perfect plasticity. The diverging relaxation time near $s=s_{y}$, however, has no simple analog in conventional descriptions of strain hardening.

This one-parameter fit (4.4) to the shape of the strain-hardening curve is much too simple even for the fully nonlinear STZ model, where the behaviors at small stresses and at stresses near $s_{y}$ are determined by different groups of parameters. Moreover, the small-s behavior of this $t \rightarrow \infty$ curve is not what is measured experimentally. In real materials and in the full STZ theory, the plastic deformation rates at small stresses are too small to be observed, and the material behaves as if it were purely elastic. For an illustration of this behavior, see Fig. 5 in [2].

For $s>s_{y}, \Delta$ goes to $1 / \gamma s$ and

$$
\dot{\varepsilon}_{\theta \theta}^{p l} \rightarrow \frac{\lambda}{\tau s}\left(s^{2}-s_{y}^{2}\right) \cong \frac{2 \lambda}{\tau}\left(s-s_{y}\right)
$$

In dynamic situations at large stress, therefore, the STZ model looks like a Bingham plastic. In short, even this highly simplified version of the STZ model describes much of both static and dynamic plasticity.

\section{B. Dynamically growing hole}

We return now to the circle problem. As a first investigation, we have numerically integrated (2.10)-(2.12), supplemented by (4.2), to find the time evolution of $s(r, t), \Delta(r, t)$, and $R(t)$. In Figs. 11 and 2, we show what happens if we suddenly apply the stress $s(r, 0)=\sigma_{\infty} R^{2}(0) / r^{2}$. We set $\lambda s_{y}=0.005, s_{y}=0.1 \mu$, and $\sigma_{\infty}=0.2 \mu$ and assume a previously undeformed system, $\Delta(r, 0)=0$. For these values of the parameters, the hole grows for a while and then stops, and a plastic zone with $s \cong s_{y}, \Delta \cong \lambda s_{y}$ forms around it. Apart from the fact that the outer boundary of the plastic zone is smooth rather than sharply defined, this static limit of the time-dependent deformation is qualitatively consistent with conventional, time-independent plasticity theory.

In Fig. 3, we show an analogous set of curves for a substantially larger value of $\lambda$, specifically, $\lambda s_{y}=0.5$. According to (4.4), this system deviates appreciably from perfect plasticity. A plastic zone does form around the hole, but it has no sharp outer boundary. If we estimate the position of this boundary, say, by finding the point of inflection in the final curve $s(r)$, we find that the relation (3.1) is strongly violated.

As in the Bingham model discussed in Section III, the STZ model predicts the existence of unbounded failure modes for sufficiently large $\sigma_{\infty}$. The stationary states of the kind shown in Figs. 1 1 and 3 cease to exist beyond $\sigma_{\infty}$. Indeed, as we show in Fig. (1) the radius of the hole diverges at $\sigma_{\infty}^{t h}$. We find these dynamically growing states via the scaling analysis of Eqs. (2.13) and (2.14). It is useful to make the following changes of variables:

$$
\frac{R^{2}(t)}{r^{2}}=\frac{1}{\xi^{2}}=\zeta ; \quad s(r, t)=s_{y} \psi(\zeta) ; \quad \Delta(r, t)=\lambda s_{y} \varphi(\zeta) .
$$

We find:

$$
\psi-\varphi+\frac{2(1-\nu) \omega \tau}{\mu \lambda} \zeta \frac{d \psi}{d \zeta}=\frac{\omega \tau}{\lambda s_{y}} \zeta\left[1+(1-2 \nu) \frac{s_{y}}{\mu} \psi(1)\right]
$$

The equation of motion for $\Delta$, i.e. (4.2), becomes:

$$
2 \omega \tau \zeta \frac{d \varphi}{d \zeta}=(\psi-\varphi)(1-\varphi \psi)
$$

Finally, (2.14) becomes:

$$
\sigma_{\infty}=s_{y} \int_{0}^{1} \frac{d \zeta}{\zeta} \psi(\zeta)
$$

As a first step in interpreting these equations, we compute the threshold $\sigma_{\infty}^{t h}$ by taking the limit $\omega \rightarrow 0$. To avoid unnecessary complication, we again set $\nu=1 / 2$. In this case, (2.13) and (4.9) reduce to $\psi \approx \varphi$ and, after a simple integration: 


$$
\lambda s_{y} \ln \left(\frac{1+\psi}{1-\psi}\right)+\frac{s_{y}}{\mu} \psi=\zeta .
$$

Note that, if $s_{y} / \mu \ll 1$ (which is generally true for realistic situations), then

$$
\psi(\zeta) \cong \tanh \left(\frac{\zeta}{2 \lambda s_{y}}\right)
$$

This solution exhibits a plastic zone with a smooth elastic-plastic boundary only for $\lambda s_{y} \ll 1$, in which case there is a region between $\zeta=2 \lambda s_{y}$ and $\zeta=1$ in which $\psi \approx 1$.

It is easy to compute $\psi(\zeta)$ without making the latter approximation and, via (4.10), to obtain the threshold stress $\sigma_{\infty}^{t h}$. If $\left(s_{y} / \mu\right)(1+2 \lambda \mu) \ll 1$, then

$$
\frac{\sigma_{\infty}^{t h}}{s_{y}} \approx 1+\ln \left(\frac{\mu}{s_{y}}\right)-\ln (1+2 \lambda \mu),
$$

which agrees with $(3.2)$ when $\lambda=0$ and $\nu=1 / 2$, i.e. in the limit of perfect plasticity. Note that we have chosen the parameters in Fig. 1 to lie within this range. If, on the other hand, $\lambda s_{y} \gg 1$, then $\sigma_{\infty}^{t h} \approx 1 / 2 \lambda$. Here the threshold lies below $s_{y}$; the material is highly deformable and conventional plasticity theory has no range of validity. We illustrate this point in Fig. 5 .

Expanding the solutions of (4.8) and (4.9) to first order in $\omega$, we find the following behavior near threshold: For $s_{y} / \mu \ll 2 \lambda s_{y} \ll 1$,

$$
\omega \approx \frac{2 \lambda}{\tau}\left[1+2 \lambda s_{y} \ln \left(\lambda s_{y}\right)\right]\left(\sigma_{\infty}-\sigma_{\infty}^{t h}\right)
$$

and, for $\lambda s_{y} \gg 1$

$$
\omega \approx \frac{\lambda}{\tau}\left(\sigma_{\infty}-\sigma_{\infty}^{t h}\right)
$$

In each of the last two equations, the quantity $\sigma_{\infty}^{\text {th }}$ has the value computed in the corresponding limit in the previous paragraph.

We have studied the behavior of the similarity solutions (4.8)-(4.10) numerically. Just as in the Bingham plastic, the deviatoric stress at the surface of the hole in the STZ material grows with the hole expansion rate $\omega$ as shown in Fig. 6. While intuitively obvious, this result is relevant to understanding stress transmission to brittle crack tips. In Fig. 0 we show the $\lambda$-dependence of the stress for a slowly expanding hole. This solution is essentially the $\omega \rightarrow 0$ limit obtained in Eq. (4.11). In a "softer" material with larger $\lambda$, the plastic zone shrinks and disappears completely for a large enough $\lambda$. This softening of the material for larger $\lambda$ leads to the decrease in the threshold stress $\sigma_{\infty}^{t h}$ above which dynamic failure modes exist.

\section{DISCUSSION}

Our principal point is that the STZ model, a simple example of a rate-and-state theory, provides an extremely compact and physically motivated description of essentially all of plasticity theory, both static and time dependent. In just two constitutive relations, 4.1) and (4.2), containing just two dimensionless groups of parameters, $s_{y} / \mu$ and $\lambda s_{y}$, plus one time constant $\tau$, we capture linear viscoelasticity at small stress, strain hardening at larger stress, and a dynamic transition to viscoplasticity at a yield stress. All of these properties have been described previously in [2] for homogeneous situations and in [10] for an inhomogeneous one dimensional case.

In this paper, our principal interest has been to make contact with conventional theories of plasticity by looking at deformation near a circular hole. As a rule, we recover conventional results when both $s_{y} \ll \mu$ and $\lambda s_{y} \ll 1$. The first of these conditions, that the yield stress must be much less than the shear modulus, is generally true for ordinary materials. The second condition, according to (4.4), says that the plastic strain at which large-scale deformations start to occur must be much smaller than unity. This too is ordinarily satisfied by rigid solids. However, it remains to be seen whether this $\lambda$-dependent condition might be modified in more realistic parameterizations of rate-and-state theories.

One place where the rate-and-state theory differs qualitatively from conventional plasticity is in the interpretation of the strain hardening curve (4.4). Here, this curve must be interpreted as the $t \rightarrow \infty$ limit of the dynamic 
inelastic response to an externally applied stress, not as an instantaneous response that is mathematically equivalent to nonlinear elasticity. Moreover, the parameter $\lambda$ that determines the deviation from perfect plasticity in (4.4) is the same parameter that appears in the viscoplastic law (4.6), and is also the same parameter that determines both the shape of the plastic zone and the threshold $\sigma_{\infty}^{t h}$ at which static solutions give way to time-dependent, unbounded failure modes.

Perhaps the single most important advantage of the STZ theory is that the material in the plastic zone is characterized not just by the stress and corresponding displacement fields but also by the state variable $\Delta$. This variable tells us in a natural way that the material inside the zone will respond differently to subsequent changes in stress than will the undeformed material outside it. To take advantage of this feature, however, we must go beyond the truncated version of the STZ model that we have used here and include the strongly nonlinear, $s$-dependent rate factor derived in [2].

Without doing any further calculations, we can see how the nonlinear rate factor produces memory effects. Suppose that we start in a stressed configuration such as the one shown in Figs. 1 and 2 in which a plastic zone has formed around the hole; and suppose that we then unload the system by quickly reducing the external stress to zero. At $s=0$, the rate factor becomes extremely small; in the absence of a stress that can induce transitions from one orientation to another, the zones remain as they were in the previously stressed state. That is, $\Delta(r)$ remains unchanged on unloading. The system relaxes elastically, but the plastic degrees of freedom do not revert to their earlier values. Accordingly, there must be residual stresses in the region near the hole. The material will "remember" the magnitude and direction of its previous loading because it "knows" the function $\Delta(r)$, which will determine via the nonlinear generalizations of the constitutive equations (4.1) and (4.2) what happens when new stresses are applied.

The other feature that we have emphasized in this analysis is the dynamic failure that occurs at $\sigma_{\infty}^{\text {th }}$, a feature that also occurs in the conventional time-dependent theories. We believe that the dynamic growth modes may provide a clue for solving a long-standing puzzle in the theory of fracture, specifically, the question of how breaking stresses can be transmitted through plastic zones to crack tips. This puzzle pertains to quasi-static crack advance, which would seem to be impossible if, as in conventional plasticity theory, the stresses near a crack tip are constrained to be less than or equal to the yield stress. The new modes, especially those that occur when there is appreciable deviation from perfect plasticity, raise the possibility that, like the growing hole, crack growth could occur via plastic flow near the tip. We hope to explore this possibility in a subsequent report.

\section{ACKNOWLEDGMENTS}

We thank Zhigang Suo for suggesting that we study the hole problem as a way to understand the issues raised in this paper. This research has been supported primarily by U.S. DOE Grants DE-FG03-84ER45108 and DE-FG0399ER45762, and in part by the MRSEC Program of the NSF under award number DMR96-32716.

[1] E. W. Hart, Acta Metall. 18, 599 (1970).

[2] M. L. Falk and J. S. Langer, Phys. Rev. E 57, 7192 (1998).

[3] A. Ruina, J. of Geophys. Res. 88, 10359 (1983).

[4] J. H. Dieterich, J. of Geophys. Res. 99, 2601 (1994).

[5] J. M. Carlson and A. A. Batista, Phys. Rev. E 53, 4153 (1996).

[6] F. A. McClintock, J. Appl. Mech. 35, 363 (1968).

[7] J. N. Johnson, J. Appl. Phys. 52, 2812 (1981).

[8] M. M. Carroll and A. C. Holt, J. Appl. Phys. 43, 1626 (1972).

[9] S. R. Bodner and Y. Partom, J. Appl. Mech. 39, 751 (1972).

[10] A. E. Lobkovsky and J. S. Langer, Phys. Rev. E 58, 1668 (1998).

[11] J. Lubliner, Plasticity theory (Macmillan, New York, 1990), Chap. 4.

[12] R. Hill, The mathematical theory of plasticity (Clarendon Press, Oxford, 1960), Chap. V. 


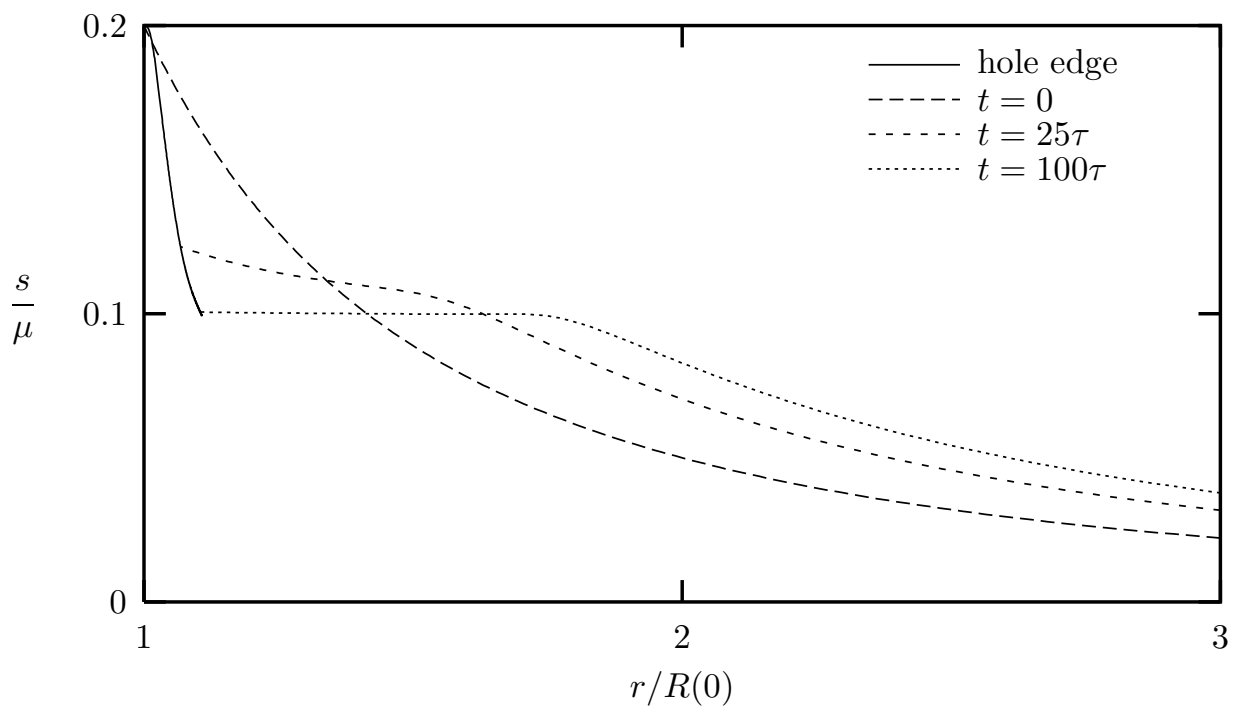

FIG. 1. Deviatoric stress $s / \mu$ as a function of the distance from the center of the hole shown at several times after the application of the stress $\sigma_{\infty}=0.2 \mu$. The yield stress $s_{y}=0.1 \mu$, Poisson's ratio $\nu=0.3, \lambda s_{y}=0.005$.

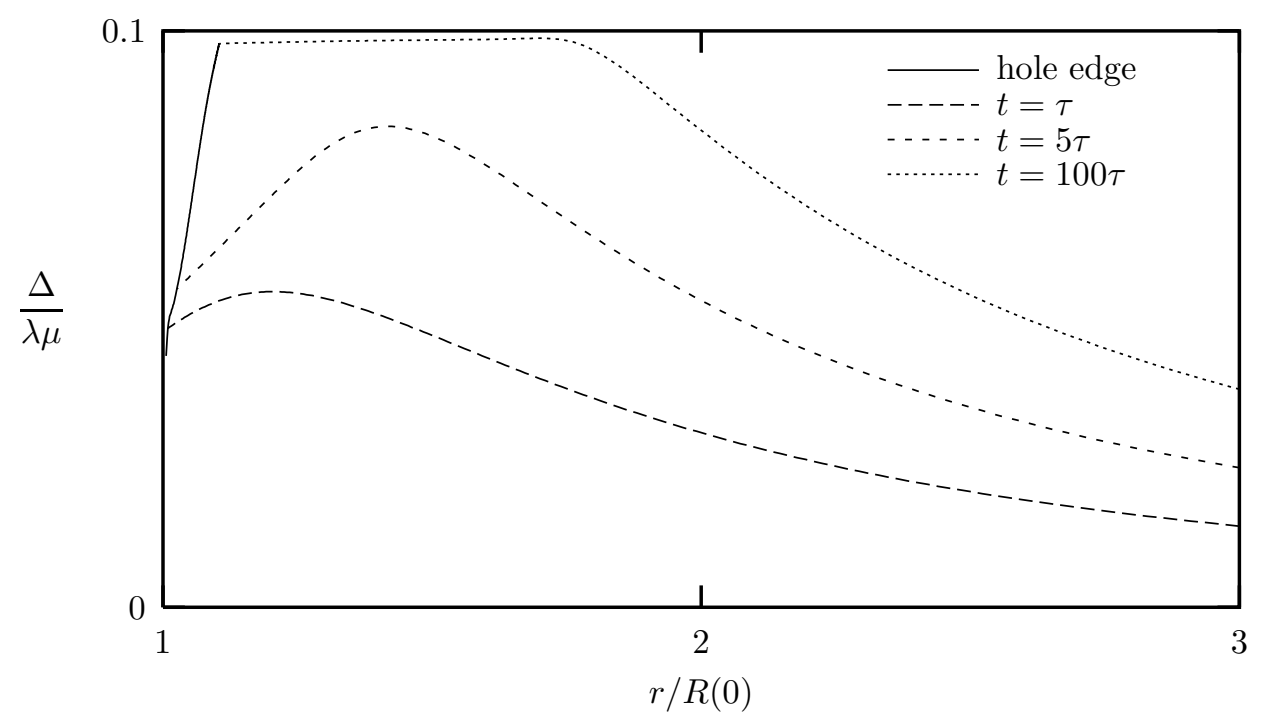

FIG. 2. Time evolution of $\Delta$ for the same values of the parameters as in Fig. 1 . 


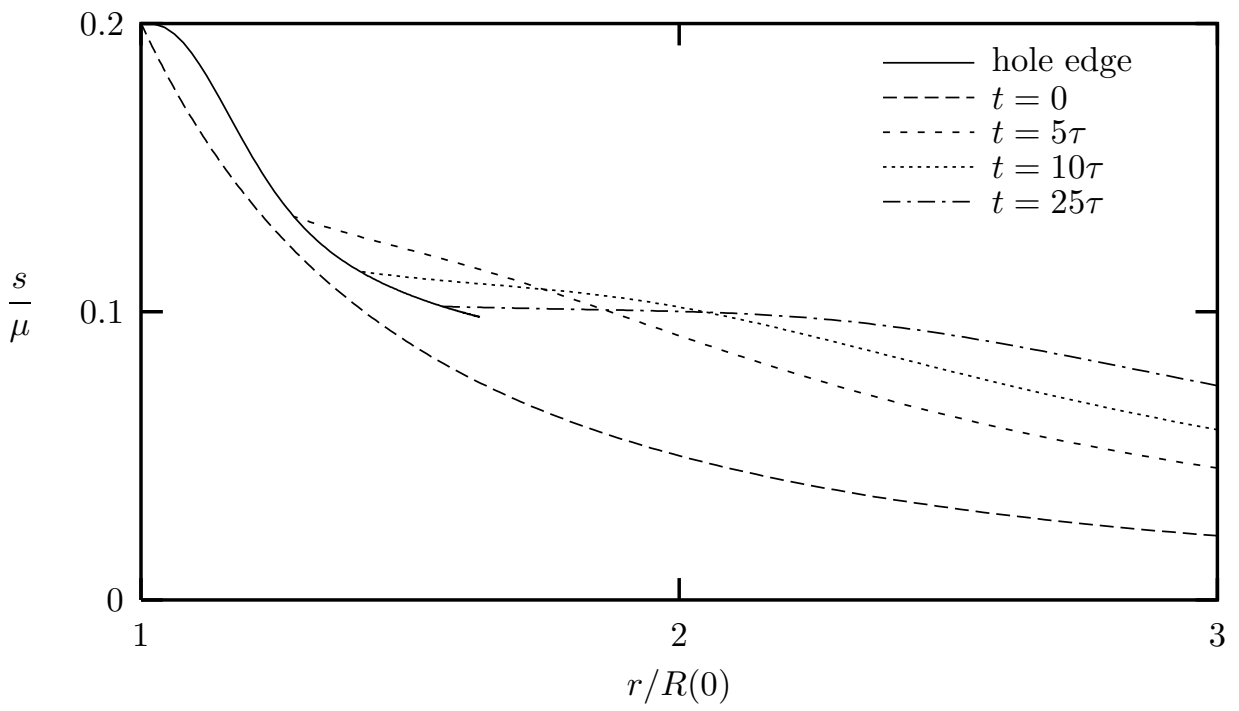

FIG. 3. Same as in Fig. 1 but wuth $\lambda s_{y}=0.05$. Note that the approach to a stationary state is faster and that the plastic zone is much less pronounced.

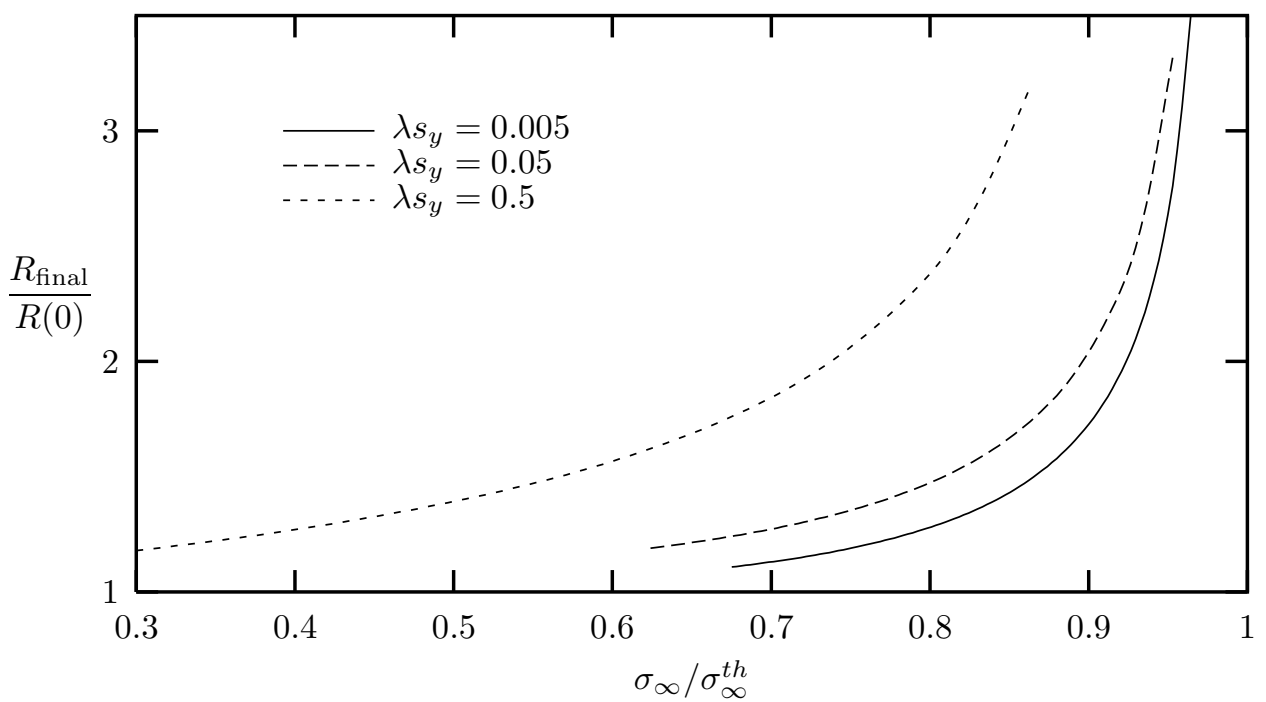

FIG. 4. The final radius of the hole versus the applied stress in units of the corresponding threshold stress for three values of $\lambda s_{y} . s_{y}=0.1 \mu$. The solid line is the prediction for the Tresca plastic. 


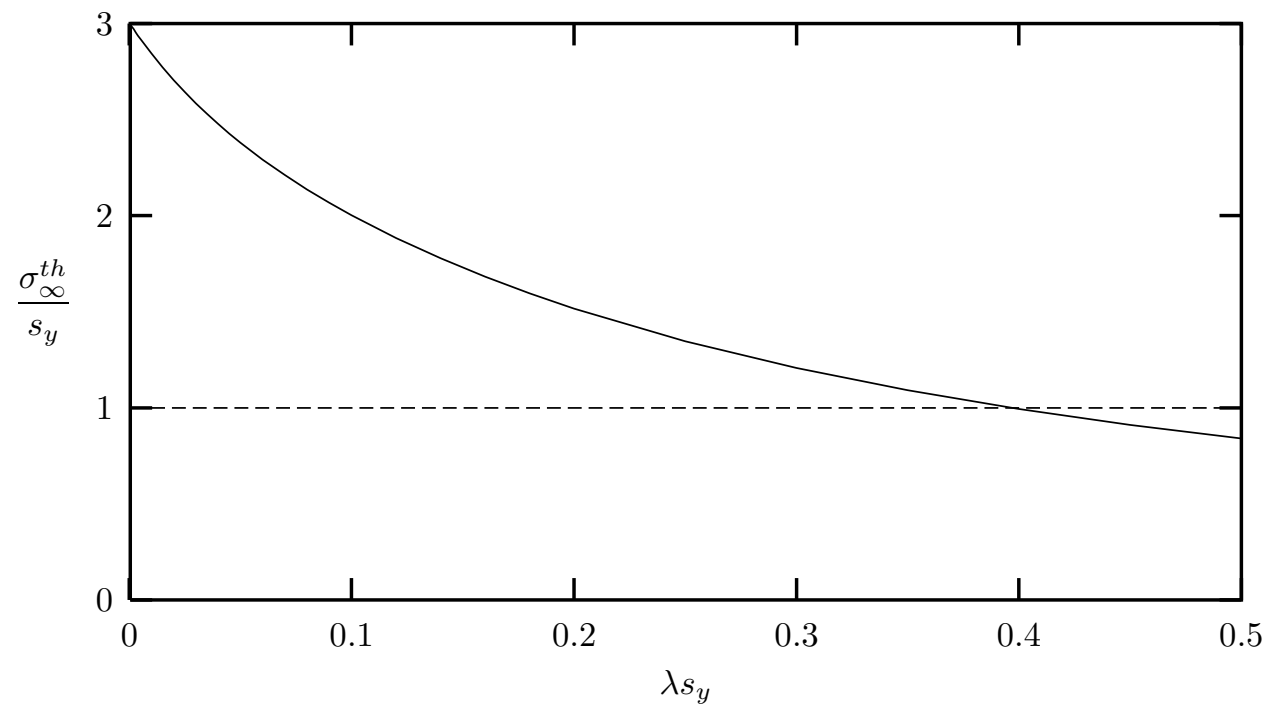

FIG. 5. Threshold stress in units of the yield stress as a function of $\lambda$. Note that for soft materials, the thresold for unbounded failure is smaller than the yield stress.

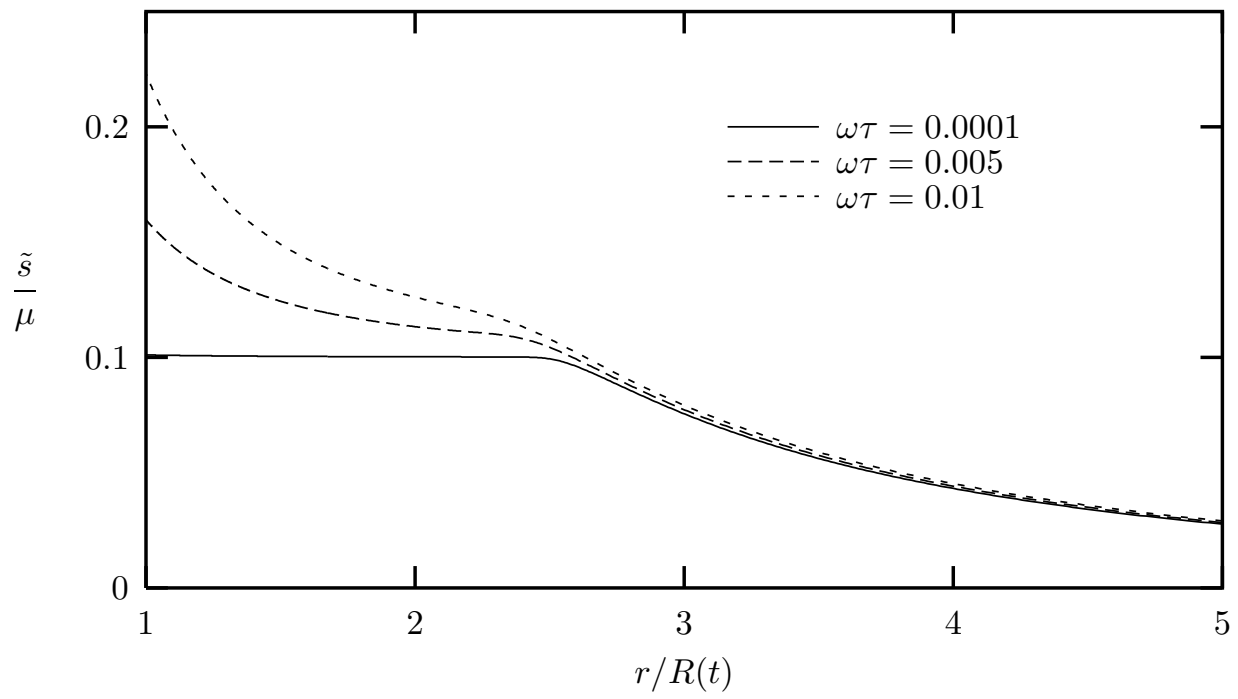

FIG. 6. Similarity solutions $\tilde{s}(r / R(t))$ for three different values of the hole growth rate $\omega . \lambda s_{y}=0.005, s_{y}=0.1 \mu$. 


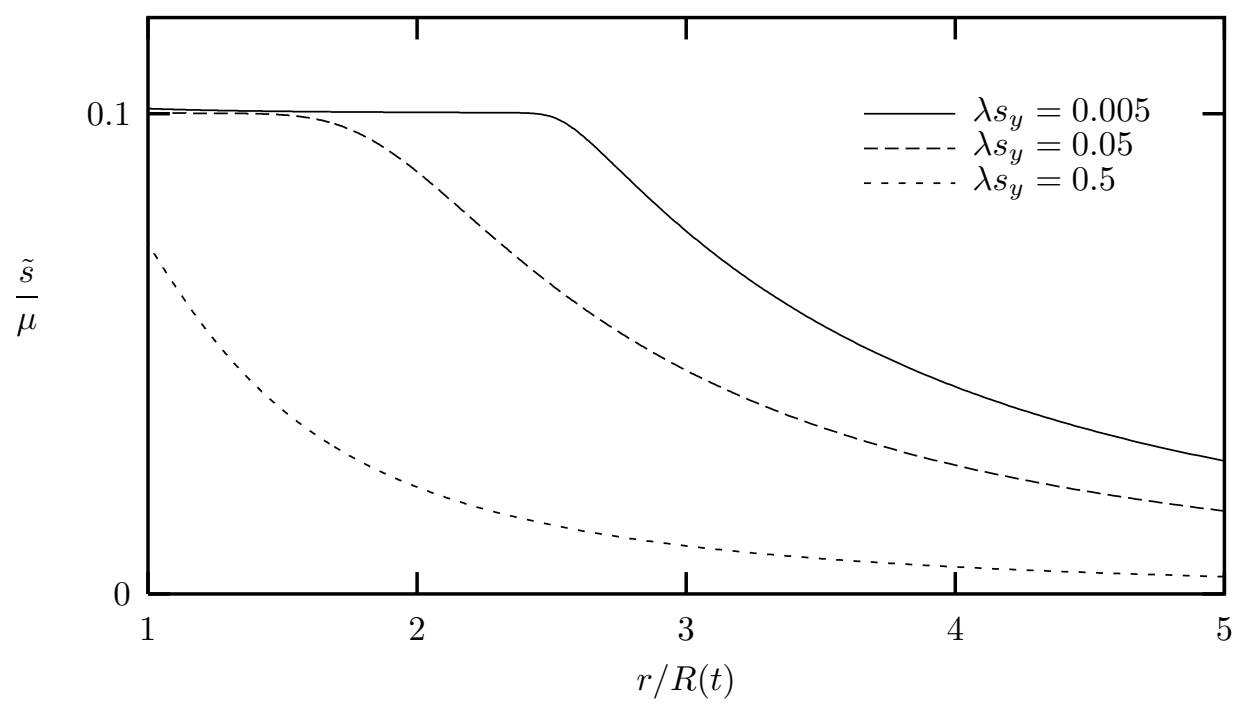

FIG. 7. Similarity solutions $\tilde{s}(r / R(t))$ for three different values of $\lambda s_{y}$ in the case of a small growth rate $\omega \tau=0.0001$. 